\title{
RESEARCH
}

Open Access

\section{Effects of long-term sleep disruption on cognitive function and brain amyloid- $\beta$ burden: a case-control study}

Jana Thomas ${ }^{1,2,3^{*}}$ (D), Sharon J. Ooms ${ }^{2,3}$, Lara J. Mentink ${ }^{1,2,3}$, Jan Booij ${ }^{4,5}$, Marcel G. M. Olde Rikkert ${ }^{1,2,3}$, Sebastiaan Overeem ${ }^{6,7 \dagger}$, Roy P. C. Kessels ${ }^{2,3,8+}$ and Jurgen A. H. R. Claassen ${ }^{1,2,3^{*}}$

\begin{abstract}
Background: Recent evidence indicates that disrupted sleep could contribute to the development of Alzheimer's disease by influencing the production and/or clearance of the amyloid- $\beta$ protein. We set up a case-control study to investigate the association between long-term work-induced sleep disruption, cognitive function, and brain amyloid- $\beta$ burden.

Methods: Nineteen male maritime pilots (aged 48-60 years) with chronic work-related sleep disruption and a sex-, age-, and education-matched control sample ( $n=16$, aged 50-60 years) with normal sleep completed the study. Primary sleep disorders were ruled out with in-lab polysomnography. Additional sleep measurements were obtained at home using actigraphy, sleep-wake logs, and a single-lead EEG device. Cognitive function was assessed with a neuropsychological test battery, sensitive to early symptomatic Alzheimer's disease. Brain amyloid- $\beta$ burden was assessed in maritime pilots using ${ }^{18}$ F-flutemetamol amyloid PET-CT.

Results: Maritime pilots reported significantly worse sleep quality (Pittsburgh Sleep Quality Index (PSQI) $=8.8 \pm 2.9$ ) during work weeks, compared to controls (PSQI $=3.2 \pm 1.4 ; 95 \% \mathrm{Cl} 0.01$ to $2.57 ; p=0.049$ ). This was confirmed with actigraphy-based sleep efficiency ( $86 \% \pm 3.8$ vs. $89.3 \% \pm 4.3 ; 95 \% \mathrm{Cl} 0.43$ to $6.03 ; p=0.03$ ). Home-EEG recordings showed less total sleep time (TST) and deep sleep time (DST) during work weeks compared to rest weeks (TST 318.56 (250.21-352.93) vs. TST 406.17 (340-425.98); $p=0.001$; DST 36.75 (32.30-58.58) vs. DST 51.34 (48.37-69.30); $p=0.005)$ ). There were no differences in any of the cognitive domains between the groups. For brain amyloid- $\beta$ levels, mean global cortical standard uptake value ratios of ${ }^{18} \mathrm{~F}$-flutemetamol were all in the normal range $(1.009 \pm 0.059 ; 95 \% \mathrm{Cl} 0.980$ to 1.037$)$, confirmed by visual reads.
\end{abstract}

Conclusions: Capitalizing on the particular work-rest schedule of maritime pilots, this study with a small sample size observed that long-term intermittent sleep disruption had no effects on global brain amyloid- $\beta$ levels or cognitive function.

Keywords: Sleep disruption, Shift work, Alzheimer's disease, Amyloid- $\beta$, Cognitive function

\footnotetext{
* Correspondence: Jana.Thomas@radboudumc.nl:

Jurgen.Claassen@radboudumc.nl

${ }^{\dagger}$ Sebastiaan Overeem and Roy P. C. Kessels contributed equally to this work.

'Department of Geriatric Medicine, Radboud University Medical Center, 6525,

GC, Nijmegen, The Netherlands

Full list of author information is available at the end of the article
}

(c) The Author(s). 2020 Open Access This article is licensed under a Creative Commons Attribution 4.0 International License, which permits use, sharing, adaptation, distribution and reproduction in any medium or format, as long as you give appropriate credit to the original author(s) and the source, provide a link to the Creative Commons licence, and indicate if changes were made. The images or other third party material in this article are included in the article's Creative Commons licence, unless indicated otherwise in a credit line to the material. If material is not included in the article's Creative Commons licence and your intended use is not permitted by statutory regulation or exceeds the permitted use, you will need to obtain permission directly from the copyright holder. To view a copy of this licence, visit http://creativecommons.org/licenses/by/4.0/. The Creative Commons Public Domain Dedication waiver (http://creativecommons.org/publicdomain/zero/1.0/) applies to the data made available in this article, unless otherwise stated in a credit line to the data. 


\section{Background}

Sleep loss has been associated with increased risk of dementia in later life, specifically dementia caused by Alzheimer's disease (AD). In a meta-analysis of 27 studies with nearly 70.000 participants, sleep loss-mostly defined as self-reported sleep of $<6 \mathrm{~h}$ per night-carried an average relative risk of 1.68 (95\% CI 1.45 to 1.86$)$ of developing dementia caused by $\mathrm{AD}$ [1]. This finding is relevant, because the etiology of late-onset $\mathrm{AD}$ remains unknown and therapeutic options are limited, making sleep a potential target for prevention or treatment of $\mathrm{AD}[2,3]$. The association between sleep loss and AD could be explained by reverse causality, wherein sleep loss is an early, preclinical manifestation of Alzheimer's pathology [4-6]. However, the association may also be causal, wherein sleep loss contributes to the development of the disease. The latter hypothesis is based on a small number of animal and human studies that have identified mechanisms that could explain how sleep loss may increase the risk of AD. In mouse models of genetic and sporadic AD, for example, sleep loss increased brain amyloid- $\beta$ accumulation [7-10]. In humans, we have previously showed that a single night of full sleep deprivation impaired the overnight reduction in CSF amyloid- $\beta$, causing $10 \%$ higher levels of CSF amyloid- $\beta$ the next morning [11]. Also in humans, selective disruption of slow wave sleep (SWS), without affecting other sleep stages, led to a comparable overnight difference in CSF amyloid- $\beta$ [12]. Additionally, acute increases (5\%) in PET-amyloid- $\beta$ levels in the hippocampus and thalamus were observed after a single night of full sleep deprivation [13]. Two mechanisms have been proposed to explain the relationship between sleep loss and amyloid- $\beta$ accumulation. First, the clearance of soluble toxic waste (including amyloid- $\beta$ ) from the central nervous system, characterized by exchange of interstitial and cerebrospinal fluids through the glymphatic pathway, appears more effective during sleep than in wakefulness [14-18]. Second, the production of amyloid- $\beta$ may be increased during wakefulness and reduced during sleep (especially SWS) $[5,11,17,19,20]$. These findings have led to the hypothesis that long-term sleep loss, through repetitive episodes of amyloid- $\beta$ accumulation, may contribute to AD. However, evidence from human studies is lacking, and it remains unknown which quantity of sleep loss, both in terms of duration and intensity, would be required to raise the risk of developing dementia due to AD.

The unique work of maritime pilots in the Netherlands offers an opportunity to study the association between long-term sleep disruption and AD risk. Maritime pilots have a work schedule characterized by one week of irregular and unpredictable working hours, leading to reduced and fragmented sleep, followed by a rest week with unrestricted sleep. Their sleep disruptions are caused by external, occupational factors, which reduce the bias of intrinsically caused sleep problems that could represent an early symptom preceding the clinical manifestation of dementia due to $\mathrm{AD}$ (i.e., reverse causality). In this group of maritime pilots, we sought to explore the effects of long-term, externally induced sleep disruption on cognitive function and brain amyloid- $\beta$ burden, as biomarker of AD.

\section{Methods \\ Study design}

The SCHIP study (Sleep Cognition Hypothesis In maritime Pilots) is a case-control study in healthy volunteers under the hypothesis that repeated nights of sleep loss may contribute to the risk of dementia due to AD by gradually increasing amyloid- $\beta$ levels. The study took place between December 2016 and May 2019 and was conducted and reported according to the STROBE guidelines for case-control studies. The timing between measurements was consistent across all participants. Sample size calculations were performed using $G^{*}$ power [21] and published previously [22]. The study protocol was peer-reviewed and published in BMJ Open [22].

\section{Study population}

We included 19 middle-aged (mean age $=53$; age range: 48 to 60 years) men from the national organization of Dutch maritime pilots (Nederlandse Loodswezen). The profession of maritime pilots in the Netherlands is almost exclusively (99\%) male. Their profession is characterized by sleep disruptions caused by external, occupational factors, wherein every other week is characterized by sleep disruption. We recruited maritime pilots with a work history of an average of 20 years (mean $=19.8$; range 10 to 30 years). Details of the study population and their occupation have been described in our methods paper [22] and can be found in the additional information file (see Additional file 1).

Maritime pilots were compared to age-, sex-, and education-matched healthy volunteers $(n=16$; mean age $=57$; age range 51 to 62 years) with occupations comparable in intellectual demand, but with regular working hours (no shift work). Control participants had normal sleep, confirmed by a Pittsburg Sleep Quality Index (PSQI) of $<5$ as well as regular bed times (between 8 p.m. and midnight), and regular wake-up times (between 5 a.m. and 9 a.m.). Participants were excluded from taking part in the study if they were using neuroactive medications, consumed $>30$ alcoholic beverages per week, had a body mass index of $>30 \mathrm{~kg} / \mathrm{m}^{2}$, suffered from intrinsic sleeping disorders (i.e., insomnia, REM sleep behavioral disorder; ruled out by PSG) or, for controls only, if they had self-reported cognitive complaints 
(indicated by Cognitive Failure Questionnaire (CFQ) and general health questionnaire). Vascular health was assessed during study visits, using a history of healthrelated events, i.e., high cholesterol, smoking, diabetes, and hypertension in addition to blood pressure measurements in maritime pilots. Baseline characteristics are listed in Table 1; Fig. 1 provides a study flow chart.

\section{Ethical approval}

The SCHIP study was approved by the institutional review board (CMO Region Arnhem-Nijmegen, NL55712.091.16, file number 2016-2337) and performed in accordance with good clinical practice guidelines and the world medical associations code of ethics (Declaration of Helsinki). Written informed consent was obtained from all participants after they received detailed study information. Participants received a stipend of 50 euros for participating, travel costs were compensated.

\section{Sleep measurements}

We combined several complementary methods to assess sleep quality.

\section{PSQI}

PSQI was filled in twice by maritime pilots (work and rest week) and once by controls.

\section{Actigraphy and sleep log}

Accelerometer-based sleep measurements (Actiwatch 2; Philips Respironics, Eindhoven, The Netherlands) in maritime pilots and controls were collected for a period of 10 consecutive days. Data was validated with sleepwake diaries. For maritime pilots, these days contained a mixture of work and rest days. Measurements were taken during the screening phase of the study (Fig. 1), before cognitive testing and amyloid PET scans.

\section{Polysomnography (2016/2017)}

To ascertain that neither maritime pilots nor controls had intrinsic sleep disorders, we performed full polysomnography (PSG) in a sleep lab (Kempenhaeghe, Heeze, The Netherlands), registering total sleep time (TST), sleep stages (N1, N2, N3, REM), wake time after sleep onset (WASO), sleep efficiency (SEF), and sleep onset latency (SOL). The PSG took place on Sunday nights during rest weeks for maritime pilots and normal weekend days for controls, between 2016 and 2017 (Fig. 1).

\section{Home-EEG (2019)}

We performed objective measurements of sleep quality during work and rest weeks in 13 of the 19 maritime pilots. For this, we used a novel and innovative device with a dry, single-lead EEG electrode (SmartSleep; Philips, Eindhoven, The Netherlands) [23]. This device is a headband that records EEG signals and can differentiate between light and deep sleep. Furthermore, it measures total sleep time (TST), sleep onset latency (SOL), number of short awakenings ( $<5 \mathrm{~min}$ awake), and number of arousals ( $>5 \mathrm{~min}$ awake). Maritime pilots were instructed to wear the headband for 20 consecutive days (10 workdays and 10 rest days). These measurements took place in 2019, before the amyloid PET scan (Fig. 1).

\section{Cognitive assessment}

The aim of the cognitive assessment was to explore effects of long-term exposure to sleep disruption in maritime pilots on AD-related cognitive impairment. Therefore, we applied a cognitive test battery that was designed to detect cognitive dysfunction in preclinical AD [24]. Tests focused on episodic memory (Logical Memory Subtest from the Wechsler Memory Scale Fourth Edition (WMS-IV LM), Rey Auditory Verbal Learning Test (RAVLT)), semantic memory and language (letter and semantic fluency, Boston Naming Test), working memory and executive function (Digit Span subtest from the Wechsler Adult Intelligence Scale - Fourth Edition (WAIS-IV), Trail Making Test (TMT) parts A and B, WAIS-IV Coding), and attention (Test of Attentional Performance 2.0, TAP). Overnight memory consolidation was assessed using a novel paradigm based on the Doors Test [25], assessing visual recognition memory after short delay (10 $\mathrm{min})$ and memory consolidation after long delay (after sleep). An overview about the cognitive tasks is summarized in the additional information file (see Additional file 2) and described in detail in our methods paper [22]. Cognitive tests were performed in the morning following PSG (in 2016 and 2017).

\section{Amyloid PET-CT imaging with ${ }^{18}$ F-flutemetamol}

Brain PET-CT scans were acquired in 2019 (Fig. 1) in maritime pilots only, since outcomes can be compared to normative data from the literature. We used the validated tracer ${ }^{18}$ F-flutemetamol [26], a tracer that performs comparable to ${ }^{11} \mathrm{C}$-PIB [27]. Previous studies suggested that CSF and PET measurements of amyloid- $\beta$ are in high concordance [28-31], while some suggest that PET is more powerful and more specific to $\mathrm{AD}$ pathology [32, 33]. Static brain images were acquired 90-110 min post-injection (four frames of $5 \mathrm{~min}$ ) after bolus injection of approximately $185 \mathrm{MBq}{ }^{18} \mathrm{~F}$-flutemetamol on a Siemens Biograph mCT. To measure tissue uptake ratios, PET scans of the PET-CT session were registered to the $\mathrm{CT}$ scan of the PET-CT session by rigid body linear registration with nearest neighbor interpolation using FSL's FLIRT (FMRIB's Linear Image Registration Tool) [34-36]. CT scans were then registered to the MNI152 $2 \mathrm{~mm}$ skull template by affine 


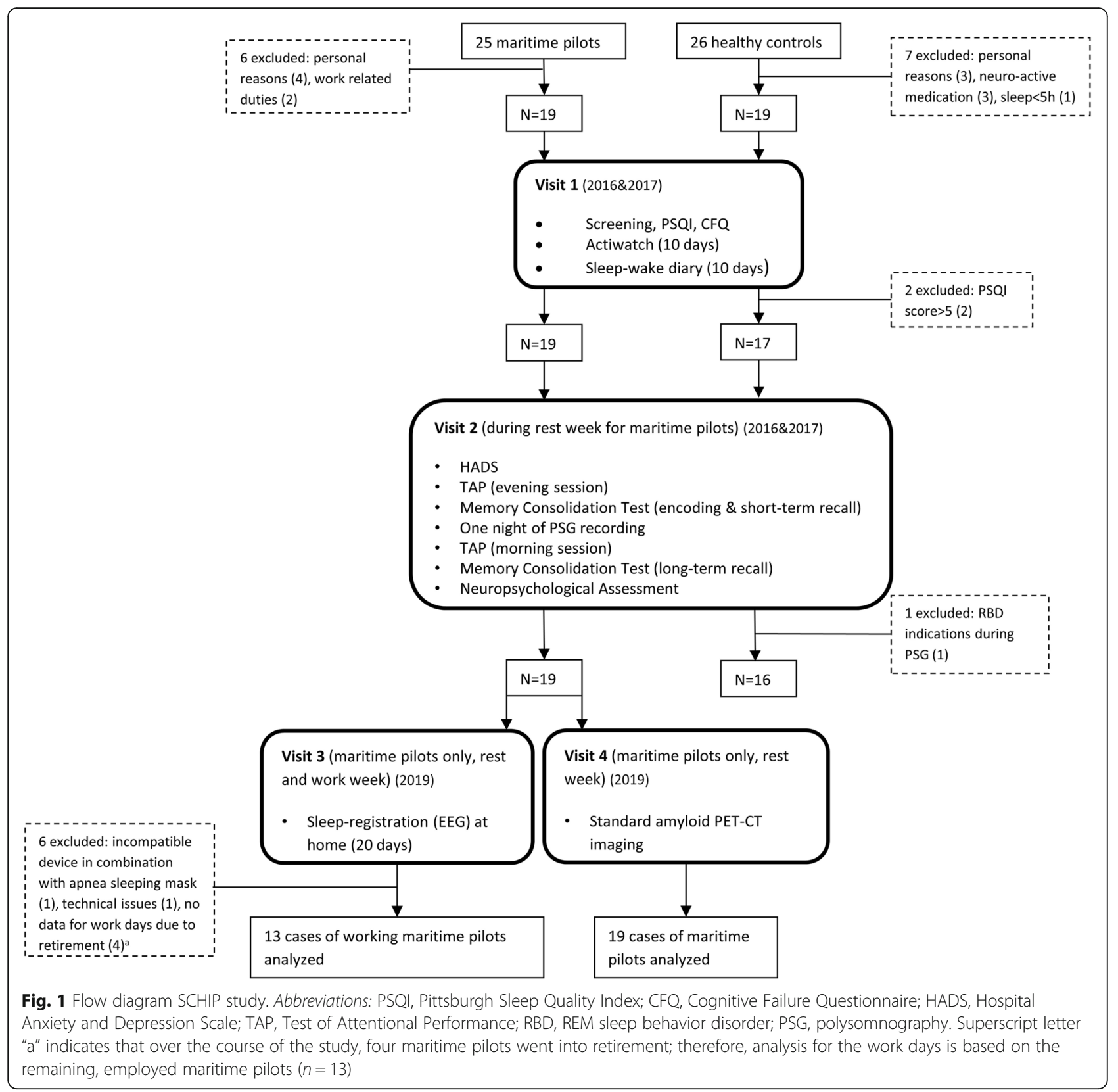

linear registration with a mutual information cost function and nearest neighbor interpolation using FSL's FLIR $\mathrm{T}$ and by non-linear registration using FSL's FNIRT (FMRIB's Non-linear Registration Tool) [34, 35]. These transformations were combined to align the PET scan to the MNI152 space in one single step. Tissue ratio was used as outcome measure, which is equivalent to the standard uptake value ratio (SUVR). The global cortical areas as well as prefrontal and temporal cortex and the cerebellum were selected with the MNI152 $2 \mathrm{~mm}$ cortical atlas. Subsequently mean uptake values of these regions of interest (ROIs) and the tissue ratio, equivalent to the SUVR, were calculated using the cerebellum as reference region [37, 38]. Normal global SUVR in a cognitively healthy population (aged 3060) was $1.3( \pm 0.09)$ [27], comparable to mean SUVR of $1.29( \pm 0.2)$ reported by Thurfjell et al. [39]. As additional step, all scans were visually rated as positive/negative for the presence of amyloid- $\beta$ deposition by an experienced and trained [40] nuclear medicine physician (JB) using validated criteria [37].

In the original protocol, an additional MRI scan was planned for PET-MRI co-registration to allow more detailed regional analyses of amyloid- $\beta$ uptake [22]. Because of limitations in funding, this could not be performed for this study after all. 


\section{Statistical analysis}

Statistical analyses were performed using IBM SPSS Statistics for Windows, version 20.0 (IBM Corp., Armonk, NY, USA). Alpha was set at 0.05 and tested two-sided. All continuous variables were assessed for normal distribution by inspection of histograms and the Shapiro-Wilk test. Normally distributed data are shown as mean $\pm \mathrm{SD}$. Not normally distributed data are presented with median and interquartile ranges (IQR). For primary outcomes of cognitive assessment, raw test scores were transformed into $z$-scores for each neuropsychological test. Z-scores were computed in SPSS and were based on mean and SD of the whole sample. For the other primary outcome measure, amyloid- $\beta$ burden, we used the mean global standard value uptake ratio (SUVR) and the dichotomous visual read of the amyloid PET scans (positive/negative) [37]. An independent samples $t$-test was performed to compare normally distributed outcome measures between the groups (shown as mean $\pm \mathrm{SD}$ ); not normally distributed outcome measures were assessed with Mann-Whitney $U$ tests (shown as median (IQR)). With regard to the home-EEG data, a Wilcoxon signed rank test was performed to compare deep sleep time (DST) during work weeks to DST during rest periods.

\section{Results}

After exclusion and drop-out of participants (see Fig. 1), 19 maritime pilots and 16 controls completed the study (Fig. 1, Table 1).

Maritime pilots were on average 4 years younger than controls (Table 1; 95\% CI -6.139 to -1.716 ). Results from the independent $t$-test did not indicate other

Table 1 Baseline characteristics

\begin{tabular}{lll}
\hline Characteristics & Controls, $n=16$ & Maritime pilots, $n=19$ \\
\hline Age, years & $57 \pm 2.9$ & $53 \pm 3.4$ \\
Educational attainment, years & $17.4 \pm 7.3$ & $18 \pm 0$ \\
BMl, kg/m² & $25.5 \pm 2.7$ & $25.7 \pm 2.7$ \\
History of diabetes & $0(0)$ & $0(0)$ \\
SBP, $\mathrm{mmHg}$ & $\mathrm{NA}$ & $148.0 \pm 16.4$ \\
DBP, mmHg & $\mathrm{NA}$ & $90.16 \pm 11.7$ \\
Medication ("yes/no") & $2(10.5)$ & $4(21.1)$ \\
Smoking ("yes/no") & $3(15.8)$ & $3(15.8)$ \\
History of hypertension & $0(0)$ & $0(0)$ \\
History of high cholesterol & $0(0)$ & $1(5.3)$ \\
CFQ & $26.4 \pm 10.8$ & $29 \pm 7.8$ \\
HADS Anxiety & $4.8 \pm 3$ & $4.0 \pm 1.7$ \\
HADS Depression & $3.6 \pm 2.5$ & $3.7 \pm 2.7$
\end{tabular}

Data is shown as mean \pm SD or no. (\%) (for normally distributed data) Abbreviations: $B M I$ body mass index, $S B P$ systolic blood pressure, $D B P$ diastolic blood pressure, CFQ Cognitive Failure Questionnaire, HADS Hospital Anxiety and Depression Scale, NA not applicable differences between the groups at baseline (Table 1). All participants were Dutch, of white European descent, and had the same level of education.

\section{Sleep characteristics \\ PSQI}

Maritime pilots reported worse sleep quality on the PSQI compared to controls, during rest weeks but especially during work weeks (Table 2). When comparing PSQI scores between work week and rest week within maritime pilots, results of the $t$-test revealed that the average PSQI score for work weeks was almost twice the score for rest weeks, with values exceeding the validated cutoff point $(\geq 7)$ for abnormal sleep behavior (Table 2).

\section{Actigraphy}

Subjective reports (PSQI) of poor sleep was confirmed by data from 10 days of actigraphy (mix of workdays and rest days), which indicated more awakenings and less sleep efficiency in the maritime pilot group compared to controls (Table 2).

\section{PSG (2016/2017)}

Both maritime pilots and controls had normal sleep patterns, including normal amount of DST (Table 2), ruling out intrinsic sleep disorders and indicating undisturbed sleep in maritime pilots during rest days.

\section{Home-EEG (2019)}

We were able to analyze EEG-based sleep measurements during work and rest days in 13 maritime pilots (of the $n=19$ maritime pilots, 4 had retired by 2019 when these measurements were scheduled, and could therefore no longer be measured during workdays; 2 could not be analyzed due to technical issues) (Fig. 1). Maritime pilots showed less TST during work weeks compared to rest weeks $(Z=-3.18 ; p=0.001)$ as well as less DST during work weeks compared to rest weeks $(Z=-2.83 ; p=$ 0.005) (Table 3). Based on the home-EEG measurements, we created hypnograms of one maritime pilot for a work week and a rest week, illustrated in Fig. 2.

\section{Cognitive assessment}

For cognitive assessment, we transformed all raw neuropsychological test scores into $z$-scores. Results from the independent $t$-test did not indicate differences between maritime pilots and controls on tests of episodic memory (WMS-IV LM recognition, RAVLT total median). Small differences were observed on semantic memory and language, in which maritime pilots performed slightly better on the Boston Naming Test compared to controls. Performance on working memory and executive function (WAIS-IV, TMT, WAIS-IV Coding) and attention (TAP 2.0) did not differ significantly between 
Table 2 Comprehensive sleep characteristics of maritime pilots and controls

\begin{tabular}{|c|c|c|c|c|}
\hline Measures & & Controls, $n=16$ & Maritime pilots, $n=19$ & $p$ value \\
\hline \multirow[t]{8}{*}{$\overline{\mathrm{PSG}}$} & TST, min & $406 \pm 44$ & $403 \pm 51$ & 0.86 \\
\hline & $\mathrm{N} 1, \min$ & $46 \pm 18$ & $41 \pm 14$ & 0.40 \\
\hline & $\mathrm{N} 2$, min & $232 \pm 36$ & $215 \pm 36$ & 0.20 \\
\hline & DST, min & $50 \pm 25$ & $66 \pm 28$ & 0.10 \\
\hline & REM, min & $68 \pm 17$ & $79 \pm 17$ & 0.10 \\
\hline & WASO, min & $61 \pm 26$ & $53 \pm 39$ & 0.48 \\
\hline & $\mathrm{SEF}, \%$ & $85.8 \pm 7.1$ & $86.1 \pm 9.4$ & 0.91 \\
\hline & $\mathrm{SOL}$, min & $8 \pm 7$ & $11 \pm 9$ & 0.32 \\
\hline \multirow[t]{2}{*}{ Actiwatch } & No. awakenings & $33.5 \pm 11.1$ & $37.8 \pm 10.3$ & 0.24 \\
\hline & $\mathrm{SEF}, \%$ & $89.3 \pm 4.3$ & $86 \pm 3.8$ & $0.03^{*}$ \\
\hline PSQI (rest week vs. control & Overall score & $3.2 \pm 1.4$ & $4.5 \pm 2.2^{*}$ & $0.049^{*}$ \\
\hline PSQI (work week vs. control) & Overall score & $3.2 \pm 1.4$ & $8.8 \pm 2.9^{* *}$ & $<0.001^{* *}$ \\
\hline
\end{tabular}

Data is shown as mean \pm SD (for normally distributed data)

Actiwatch data and PSQI were collected in 2016 and 2017. Actiwatch data was collected for a period of 10 consecutive days; for maritime pilots, these 10 days were a mix of work and rest days. PSQI was administered twice for maritime pilots, including one work week and one rest week

Abbreviations: PSG polysomnography, TST total sleep time, DST deep sleep time, REM rapid eye movement sleep, WASO wake after sleep onset, SEF sleep

efficiency, SOL sleep onset latency, PSQI Pittsburgh Sleep Quality Index

* Significant at $p<0.05$

**Significant at $p<0.001$

the groups. Maritime pilots performed slightly better on the visual recognition memory after short delay compared to controls. Long-term memory consolidation, however, did not differ between the groups. All test scores were within normal age- and education-adjusted ranges based on available normative data (data not shown). All results can be found in Table 4 .

\section{${ }^{18}$ F-flutemetamol PET-CT}

Amyloid PET scans were administered in maritime pilots only ( $n=19$, Fig. 1$)$. SUVRs in healthy populations were reported as $1.29( \pm 0.2)$ [39] and $1.3( \pm 0.09)$ [27]. The global cortical SUVR in maritime pilots was 1.009 $( \pm 0.059 ; 95 \%$ CI 0.980 to 1.037$)$ and therefore below normal values for a cognitively healthy population in this age range [27, 39]. More specifically, we detected a SUVR of $0.860( \pm 0.098 ; 95 \%$ CI 0.813 to 0.907$)$ for frontal lobes and a SUVR of $0.996( \pm 0.06$; $95 \%$ CI 0.967 to 1.025$)$ for temporal lobes. In addition, all scans were rated negative for the presence of amyloid- $\beta$ deposition on visual reading. Figure 3 shows examples of amyloid PET images from two representative participants. There were no correlations between SUVRs and sleep quality (PSQI overall score and DST (for rest and work weeks)).

\section{Discussion}

We investigated global brain amyloid- $\beta$ levels and cognitive function in a unique population experiencing longterm sleep disruption, wherein every other week was characterized by sleep disruption due to irregular working hours.

Our main finding is that, in this relatively small, but deeply phenotyped sample, this intensity and pattern of sleep disruption was not associated with elevated brain amyloid- $\beta$ levels, nor with cognitive decline.

In previous studies, a single night of full sleep deprivation, or selective restriction of deep sleep, and chronic partial sleep fragmentation (rodents only) increased brain amyloid- $\beta$ levels [8-13]. These observations have fueled the hypothesis that repeated nights of sleep loss may contribute to the risk of dementia due to $\mathrm{AD}$ by gradually increasing amyloid- $\beta$ levels.

Table 3 Results from the home-EEG measurements (maritime pilots only)

\begin{tabular}{lllll}
\hline Measures & & Rest week, $n=13$ & Work week, $n=13$ & $p$ value \\
\hline Home EEG & $\mathrm{TST}^{\dagger}$, min & $406.17(340-425.98)$ & $318.56(250.21-352.93)$ & $0.001^{*}$ \\
& $\mathrm{DST}^{\dagger}$, min & $51.34(48.37-69.30)$ & $36.75(32.30-58.58)$ & $0.005^{*}$ \\
\hline
\end{tabular}

Data is shown as median (IQR) (for not normally distributed data)

Home-EEG recordings were performed in 2019 in maritime pilots only using a dry single-lead EEG device (Philips, Eindhoven, The Netherlands)

Abbreviations: TST total sleep time, DST deep sleep time

*Significant at $p<0.05$

${ }^{\dagger}$ Means calculated based on sleep periods within work week or rest week respectively 

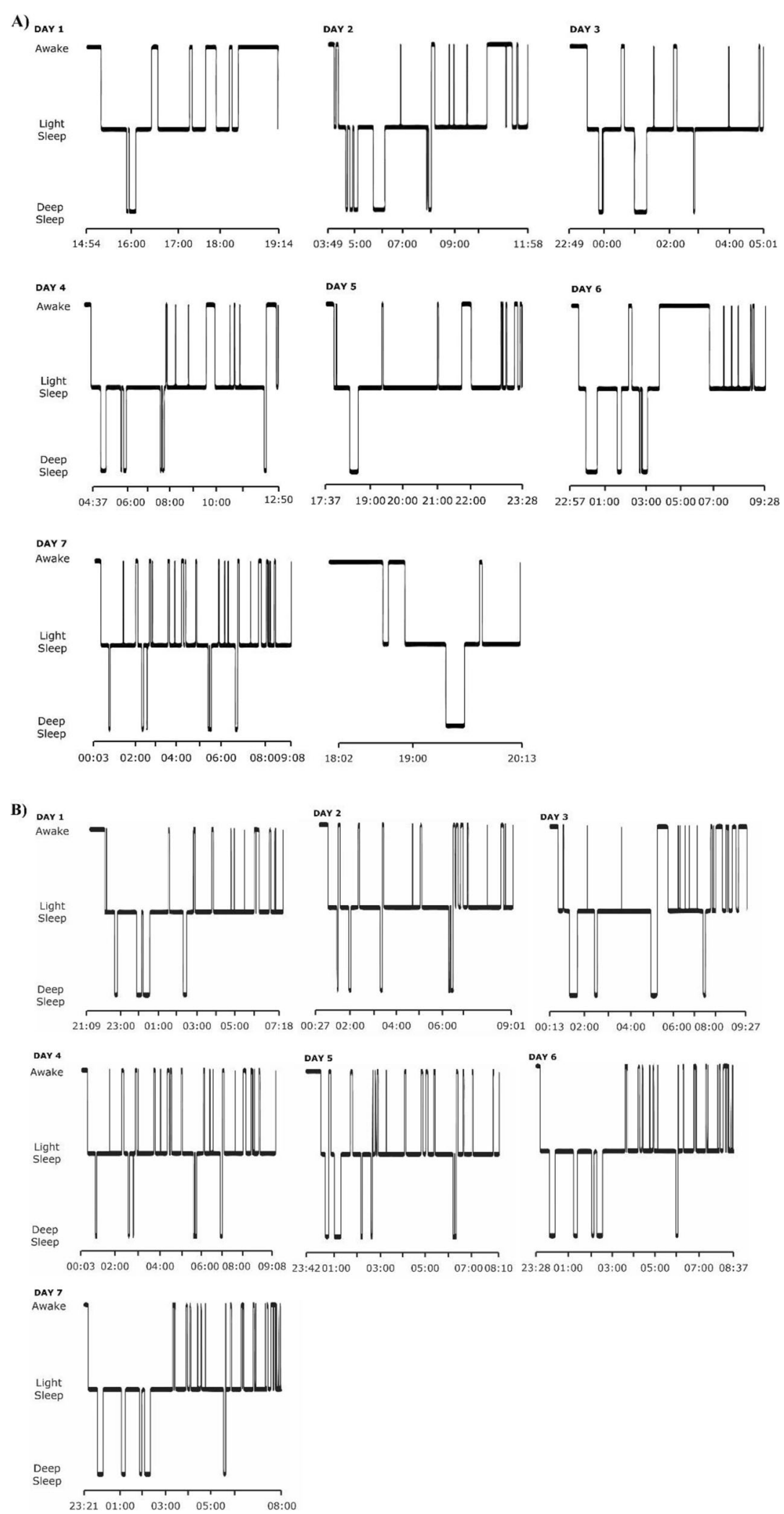

Fig. 2 Example of a maritime pilots' sleep schedule. a Hypnogram is based on 7 consecutive working days of sleep measurements with a dry electrode single-lead home-EEG device. $\mathbf{b}$ Hypnogram is based on 7 rest days of sleep measurements with a dry electrode single-lead home-EEG device 
Table 4 Results of cognitive assessment and memory consolidation

\begin{tabular}{|c|c|c|c|c|}
\hline Measures & & Controls, $n=16$ & Maritime pilots, $n=19$ & $p$ value \\
\hline \multirow[t]{3}{*}{$\overline{\text { WMS-IV }}$} & LM I & $0.16 \pm 1.07$ & $-0.08 \pm 0.99$ & 0.49 \\
\hline & LM II & $0.29(-0.93-1.01)$ & $0.11(-0.61-0.83)$ & 0.72 \\
\hline & LM recognition & $0.25 \pm 1.10$ & $-0.12 \pm 0.94$ & 0.29 \\
\hline \multirow[t]{4}{*}{ RAVLT } & Total & $-0.08(-0.63-0.61)$ & $0.77(-1.14-1.09)$ & 0.41 \\
\hline & Del. recall & $-0.08 \pm 0.76$ & $0.06 \pm 1.25$ & 0.70 \\
\hline & Del. recognition & $-0.10 \pm 1.15$ & $0.21 \pm 0.85$ & 0.37 \\
\hline & Sensitivity $A^{\prime}$ & $0.07(-0.85-0.82)$ & $0.22(-0.30-0.82)$ & 0.41 \\
\hline \multirow[t]{2}{*}{ WAIS-IV } & Coding & $-0.10 \pm 0.59$ & $0.21 \pm 1.26$ & 0.37 \\
\hline & Digit span & $-0.21 \pm 0.62$ & $0.23 \pm 1.24$ & 0.21 \\
\hline \multirow[t]{2}{*}{ TMT } & Part A & $-0.09 \pm 0.75$ & $-0.06 \pm 1.15$ & 0.94 \\
\hline & Part B & $-0.33(-0.57-0.75)$ & $-0.38(-0.96-0.50)$ & 0.24 \\
\hline \multirow[t]{3}{*}{ Fluency } & D-A-T & $0.07 \pm 0.89$ & $-0.29 \pm 1.13$ & 0.78 \\
\hline & Animal & $0.38(-0.88-0.73)$ & $0.20(-0.70-0.91)$ & 0.84 \\
\hline & Profession & $-0.26 \pm 0.85$ & $0.26 \pm 1.13$ & 0.14 \\
\hline BNT & Short version & $-0.11(-0.39-0.31)$ & $0.20(0.10-0.62)$ & $0.02^{*}$ \\
\hline \multirow[t]{2}{*}{ TAP evening } & Cued & $-0.05(-0.72-0.44)$ & $-0.39(-0.91-0.50)$ & 0.37 \\
\hline & Un-cued & $-0.05(-0.87-0.89)$ & $-0.17(-0.76-0.45)$ & 0.84 \\
\hline \multirow[t]{2}{*}{ TAP morning } & Cued & $-0.10(-0.50-0.66)$ & $-0.29(-0.71-0.12)$ & 0.27 \\
\hline & Un-cued & $-0.13(-0.63-1.09)$ & $-0.40(-0.74-0.33)$ & 0.22 \\
\hline \multirow[t]{3}{*}{ Visual recognition—short delay (10 min) } & Sensitivity, $A^{\prime}$ & $-0.27 \pm 0.90$ & $0.46 \pm 0.58$ & $0.007^{*}$ \\
\hline & Hits & $-0.33(-1.18-0.15)$ & $0.64(0.15-0.64)$ & $0.03^{*}$ \\
\hline & False alarms & $0.19 \pm 1.06$ & $-0.27 \pm 0.84$ & 0.16 \\
\hline \multirow[t]{3}{*}{ Memory consolidation-long delay (after sleep) } & Sensitivity, $A^{\prime}$ & $-0.08 \pm 0.95$ & $0.35 \pm 0.73$ & 0.14 \\
\hline & Hits & $0.50(-0.21-0.70)$ & $-0.08(-0.69-0.54)$ & 0.20 \\
\hline & False alarms & $0.15 \pm 0.90$ & $-0.41 \pm 0.76$ & 0.06 \\
\hline
\end{tabular}

Data is shown as mean \pm SD (for normally distributed data) or median (IQR) (for not normally distributed data)

Test results are expressed in $z$-scores. TAP: $z$-scores are based on median reaction-time. Visual recognition-short-term: assessed approximately 10 min after targets were presented. Memory consolidation after long-term took place after one night of sleep (approximately $10 \mathrm{~h}$ )

Abbreviations: WMS Wechsler Memory Scale, LM logical memory, RAVLT Rey Auditory Verbal Learning Test, WAIS Wechsler Adult Intelligent Scale, TMT Trail Making test, $B N T$ Boston Naming Test, TAP Test of Attentional Performance

*Significant at $p<0.05$

The sample of maritime pilots offered a unique opportunity to explore if long-term, externally induced sleep disruptions increase dementia risk in terms of $\mathrm{AD}$ related impaired cognitive function and amyloid- $\beta$ burden. Their sleep behavior is characterized by work weeks with disrupted sleep, alternating with rest weeks of unrestricted sleep. This pattern was confirmed using a combination of methods: self-reported disrupted sleep during work weeks was objectified by sleep diaries, actigraphy, and home-EEG measurements. Relatively normal sleep during rest weeks of maritime pilots was furthermore confirmed with PSG (compared to controls) and home-EEG measurements. Moreover, using PSG we were able to exclude intrinsic sleep disorders in this group, which is important because sleep loss may be an early manifestation of Alzheimer's pathology and could lead to a reverse causality association [41, 42]. To explore possible AD-related impaired cognitive performance, we applied a cognitive test battery that was chosen for its sensitivity to cognitive changes in early, preclinical $\mathrm{AD}$ [24]. On all cognitive domains, maritime pilots showed normal cognitive performance, compared not only to the control group, but also to normative values. This was also the case for overnight episodic memory consolidation, which is dependent on deep sleep $[5,43]$.

We considered that normal cognitive function would not rule out increased amyloid- $\beta$ levels, since early stages of amyloid- $\beta$ accumulation (indicated by PET or CSF) can have a long asymptomatic stage. Therefore, we performed additional global brain amyloid- $\beta$ imaging in maritime pilots. None of the maritime pilots had evidence of elevated amyloid- $\beta$ levels, with SUVR values remaining below the values established for a healthy population [27, 39]. In a recent meta-analysis, the 


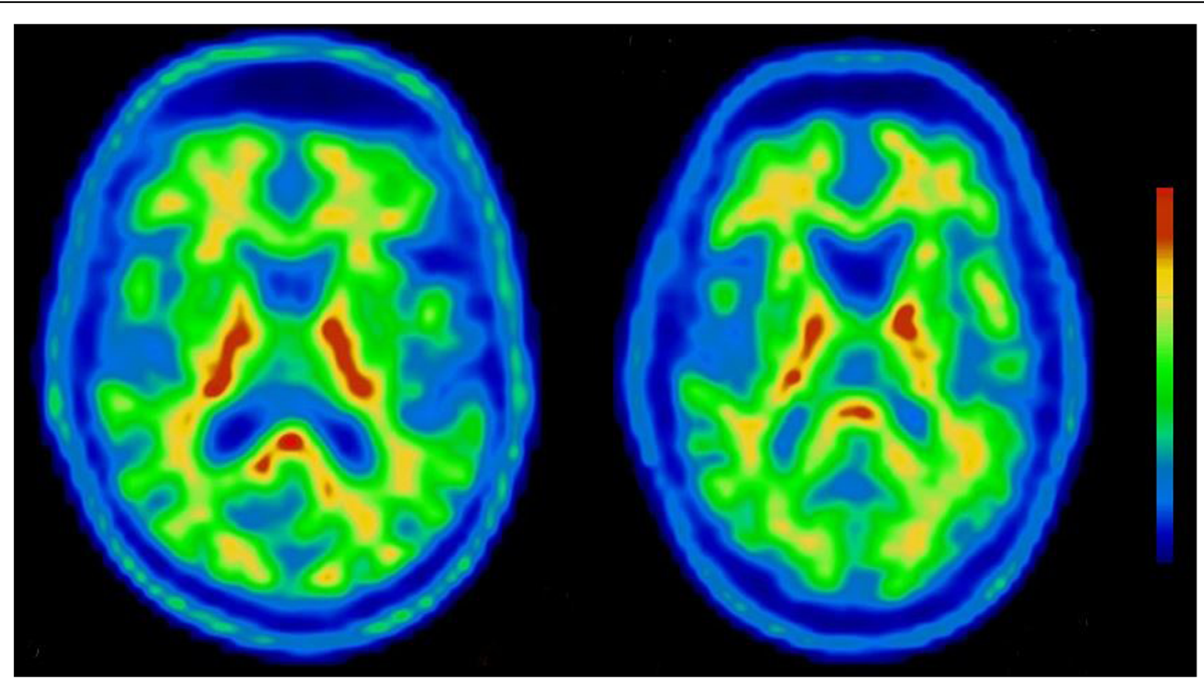

Fig. 3 Representative transversal slides from 18F-flutemetamol PET scans of two participants. Scans were acquired 90-110 min post-injection and show normal subcortical nonspecific uptake in the brain

estimated prevalence of PET amyloid-positivity in cognitively healthy men aged 55-60 years was $13 \%(95 \%$ CI 10.3 to $16 \%$ ) [44]. This indicates that our observation of a prevalence of $0 / 19$ confidently rules out elevated amyloid$\beta$ levels, even considering the relatively small sample size.

What could explain the observed absence of elevated amyloid- $\beta$ levels or impaired cognitive function, despite evidence of long-term sleep disruptions?

First, assuming that the hypothesis that sleep disruption may cause $\mathrm{AD}$ is correct, the alternating pattern of a week with unrestricted sleep following a week of disrupted sleep may be insufficient to cause elevated brain amyloid- $\beta$ levels. Either sleep disruption during $\approx 50 \%$ of nights for $\approx 20$ years is insufficient to affect amyloid- $\beta$ clearance/production or the week of normal sleep following a week of sleep disruption provides compensatory reductions in brain amyloid- $\beta$ levels. This latter option would then suggest that disrupted sleep is a modifiable risk factor and that it may not be necessary to achieve full normalization of sleep to reduce AD risk. Whether this can be extrapolated to the general population is uncertain however. The maritime pilots may, due to their profession, be better able to compensate normal sleep in their rest weeks. While most studies link reduced total sleep time $(<6 \mathrm{~h})$ to increased AD risk [1, 20 ], other work suggests that the risk is specifically linked to reduced deep sleep [12, 17]. The maritime pilots had reduced total sleep time during work weeks, but the home-EEG recordings indicate that they still achieved an average of $37 \mathrm{~min}$ of deep sleep per sleep period. Therefore, SWS may have been insufficiently impaired to result in abnormal amyloid- $\beta$ levels. This argument is, however, not supported by recent work demonstrating that a reduction in total sleep time, but not SWS, determined the increase in amyloid- $\beta$ production [20].

Second, it is possible that sleep disruption alone is insufficient to increase $\mathrm{AD}$ risk, but requires the presence of other risk factors, such as impaired glucose metabolism [45], oxidative stress [46], depression [47], or general poor vascular health [48]. Our study population was healthy and had a low vascular risk (Table 1).

Current research on this topic is still in its very early stage, with limited evidence supporting a causal relationship between sleep loss and risk of AD dementia. The association between sleep loss and $\mathrm{AD}$ may be driven by reverse causality (sleep loss as an early manifestation of $\mathrm{AD}$ ) or by a shared common pathway that causes sleep loss and increases AD risk. There is also recent evidence that found no association between sleep (subjective sleep quality) and risk of dementia [49].

Previous evidence suggesting a link between sleep and AD has been limited to a small number of studies in rodents and humans, with variations in methodology and study population selection. Furthermore, the human studies focused on the relationship between poor sleep for a short period of time (1 or 2 nights) and its effects on amyloid- $\beta$ (or tau), but have not studied actual development of AD dementia.

Longitudinal studies are available but lack rigorous assessment of sleep and biomarker evidence of AD. Our study adds information on the long-term association between poor sleep and $\mathrm{AD}$, combining objective sleep measures with established biomarkers for AD. 


\section{Strengths and limitations}

A strength of the study is the comprehensive assessments of all outcome measures: cognitive function was assessed with an extensive test battery sensitive to early, preclinical symptoms of $\mathrm{AD}$; sleep was assessed with various measurements including self-reported but also objectively measured sleep, implementing innovative techniques for sleep assessments (home-EEG); sleep disorders were ruled out using PSG; and PET-amyloid imaging was used as a validated AD biomarker. A further strength is the unique cohort of maritime pilots, with prolonged and consistent exposure to sleep loss related to their work, making this a highly valuable population that allowed us to explore poor sleep as isolated variable in relationship with the risk of $\mathrm{AD}$ dementia.

Our study is limited by the small sample size. Home-EEG measurements were available in 13 of the 19 maritime pilots. However, outcomes of these sleep measurements confirmed observations of work-related disrupted sleep based on PSQI, sleep-wake dairy and actigraphy data in the whole sample, and added novel data on total and deep sleep time during work weeks and rest weeks.

The uniqueness of the population may also cause bias. Maritime pilots are healthy, have no cardiovascular risk, and are physically active in their work, factors that may reduce their $\mathrm{AD}$ risk. They may be resilient to the consequences of sleep disruption, because they have successfully performed this work for $>10$ years. One example of such resilience could be their ability to achieve deep sleep even under conditions of fragmented and restricted total sleep during work weeks or their capacity to generate sufficient deep sleep during rest weeks.

Another limitation is that controls, although matched for sex, age, education, and general health, might not have been matched entirely with regard to personality, resilience, physical activity, or cognitive skills/general intelligence.

One could argue that the absence of tau measurements is a limitation, as recent evidence now also suggests that sleep affects tau in a similar manner as amyloid- $\beta$ [50]. We did not perform tau measurements because the maritime pilots had no evidence of cognitive impairment. Since tau pathology is strongly correlated with cognitive decline [51-53], it is highly unlikely to find evidence of tau accumulation in subjects with normal cognitive function, even more so when they are amyloid-negative. A final limitation is that amyloid- $\beta$ status was not obtained from the controls. Instead, we compared our outcomes to normative values from the literature, which were acquired with additional MRI measurements for co-registration of the amyloid PETCT scans. Since we used CT to identify global amyloid- $\beta$ instead of MRI, this difference in methodology has to be kept in mind when interpreting our results.

\section{Conclusions}

In this study, we tested the hypothesis that prolonged sleep loss increases the risk of dementia due to AD. We found that a history of work-induced, long-term sleep disruption was not associated with impairment in cognitive function, nor with elevated global brain amyloid- $\beta$ levels, in a group of healthy, middle-aged men. Taking into account the small sample size of our study, our results do not necessarily refute the hypothesis we intended to test, but neither support it. It is possible that amyloid- $\beta$ accumulation during periods with sleep disruption can be reduced in nights with normal sleep. Alternatively, sleep loss may only increase AD risk in combination with other factors. Finally, the association between sleep loss and $\mathrm{AD}$ in epidemiological studies may be driven by reverse causality. These and other hypotheses have to be tested in future studies.

\section{Supplementary information}

Supplementary information accompanies this paper at https://doi.org/10. 1186/s13195-020-00668-5.

Additional file 1. Details of the study population and their occupation. Additional file 2. Supplemental Box 1: Overview Neuropsychological Test Battery.

\section{Abbreviations}

AD: Alzheimer's disease; CFQ: Cognitive Failure Questionnaire; DBP: Diastolic blood pressure; DST: Deep sleep time; HADS: Hospital Anxiety and

Depression Scale; IQR: Interquartile range; NA: Not applicable; NRA: Number of arousals; NRI: Number of interruptions; PSG: Polysomnography;

PSQI: Pittsburgh Sleep Quality Index; RAVLT: Rey Auditory Verbal Learning Test; REM: Rapid eye movement; ROI: Region of interest; SBP: Systolic blood pressure; SCHIP: Sleep Cognition Hypothesis In maritime Pilots; SD: Standard deviation; SEF: Sleep efficiency; SOL: Sleep onset latency; SUVR: Standard uptake value ratio; SWS: Slow wave sleep; TAP: Test of Attentional

Performance; TMT: Trail Making Test; TST: Total sleep time; WAIS-IV: Wechsler Adult Intelligence Scale (4th edition); WASO: Wake after sleep onset; WMS-IV LM: Wechsler Memory Scale (4th edition) Logical Memory

\section{Acknowledgements}

We would like to thank all participants for taking part in this study and the secretary of the Dutch Maritime Pilot Association for helping with recruitment of participants. Furthermore, a special thanks to the interns who helped with data collection and to J. Doornbosch for helping with the neuropsychological test administration. Dr. T. Tsoneva and S. Pastoor from Philips helped us with data storage and raw data-extraction from the homeEEG devices. J.M.F. Liebregts provided advice on and assistance with designing graphics and figures. We would also like to thank Dr. K.V. Haak for his advice on the PET-CT imaging analyses.

\section{Authors' contributions}

JT, SJO, SO, RPCK, and JAHRC made substantial contributions to conception or design of the work. All authors (JT, SJO, LJM, JB, MGMO, SO, RPCK, JAHRC) were in charge of acquisition, analysis, and interpretation of data for the work. JT, SJO, LJM, JB, RPCK, and JAHRC were responsible for statistical data analyses. Supervision was given by JAHRC. All authors drafted the work or revised it critically for important intellectual content and approved the final version of the manuscript. All authors have agreed both to be personally accountable for their own contributions and to ensure that questions related to the accuracy or integrity of any part of the work, even ones in which the author was not personally involved, are appropriately investigated, resolved, and the resolution documented in the literature. 


\section{Funding}

This work was supported by ISAO (Internationale Stichting Alzheimer Onderzoek (now Alzheimer Nederland), grant number: 15040) awarded to JC. Philips kindly provided the home-EEG devices that were used in this study. The funders had no role in the study design or conduct; collection, analysis, or interpretation of data; nor in the writing of the report or in the decision to submit the manuscript for publication.

\section{Availability of data and materials}

Data generated and/or analyzed during this study are included in this article and its supplementary information files. Additionally, the datasets used/ analyzed in the current study are available from the corresponding author on reasonable request

\section{Ethics approval and consent to participate}

The SCHIP study was approved by the institutional review board (CMO, Commissie Mensgebonden Onderzoek, Region Arnhem-Nijmegen, NL55712.091.16, file number 2016-2337) and performed in accordance with good clinical practice guidelines and the world medical associations code of ethics (Declaration of Helsinki). Written informed consent was obtained from all participants after they received detailed study information. Participants received a stipend of 50 euros for participating; travel costs were compensated.

\section{Consent for publication}

Not applicable.

\section{Competing interests}

The authors declare that they have no competing interests.

\section{Author details}

'Department of Geriatric Medicine, Radboud University Medical Center, 6525, GC, Nijmegen, The Netherlands. Donders Institute for Brain, Cognition and Behaviour, 6525, HR, Nijmegen, The Netherlands. ${ }^{3}$ Radboud Alzheimer Centre, 6525, GA, Nijmegen, The Netherlands. ${ }^{4}$ Department of Radiology and Nuclear Medicine, Radboud University Medical Center, 6525, GC, Nijmegen, The Netherlands. ${ }^{5}$ Department of Radiology and Nuclear Medicine, Amsterdam University Medical Centers, Academic Medical Center, 1105, AZ, Amsterdam, The Netherlands. ${ }^{6}$ Sleep Medicine Centre Kempenhaeghe, 5591, VE, Heeze, The Netherlands. 'Eindhoven University of Technology, 5612, AZ, Eindhoven, The Netherlands. ${ }^{8}$ Department of Medical Psychology, Radboud University Medical Center, 6525, GA, Nijmegen, The Netherlands.

\section{Received: 21 April 2020 Accepted: 13 August 2020}

Published online: 26 August 2020

\section{References}

1. Bubu OM, Brannick M, Mortimer J, Umasabor-Bubu O, Sebastião YV, Wen Y, et al. Sleep, cognitive impairment, and Alzheimer's disease: a systematic review and meta-analysis. Sleep. 2016;40(1):zsw032.

2. Mander BA, Winer JR, Jagust WJ, Walker MP. Sleep: a novel mechanistic pathway, biomarker, and treatment target in the pathology of Alzheimer's disease? Trends Neurosci. 2016;39(8):552-66.

3. Spira AP, Gottesman RF. Sleep disturbance: an emerging opportunity for Alzheimer's disease prevention? Int Psychogeriatr. 2017;29(4):529-31.

4. Slats D, Claassen JA, Verbeek MM, Overeem S. Reciprocal interactions between sleep, circadian rhythms and Alzheimer's disease: focus on the role of hypocretin and melatonin. Ageing Res Rev. 2013;12(1):188-200.

5. Ju Y-ES, Lucey BP, Holtzman DM. Sleep and Alzheimer disease pathology [mdash] a bidirectional relationship. Nat Rev Neurol. 2014;10(2):115-9.

6. Roh JH, Huang Y, Bero AW, Kasten T, Stewart FR, Bateman RJ, et al. Disruption of the sleep-wake cycle and diurnal fluctuation of $\beta$-amyloid in mice with Alzheimer's disease pathology. Sci Transl Med. 2012;4(150): 150ra22-ra22.

7. Kang J-E, Lim MM, Bateman RJ, Lee JJ, Smyth LP, Cirrito JR, et al. Amyloid- $\beta$ dynamics are regulated by orexin and the sleep-wake cycle. Science (80-). 2009;326(5955):1005-7

8. Minakawa EN, Miyazaki K, Maruo K, Yagihara H, Fujita H, Wada K, et al. Chronic sleep fragmentation exacerbates amyloid $\beta$ deposition in Alzheimer's disease model mice. Neurosci Lett. 2017;653:362-9.
9. Rothman SM, Herdener N, Frankola KA, Mughal MR, Mattson MP. Chronic mild sleep restriction accentuates contextual memory impairments, and accumulations of cortical $A \beta$ and pTau in a mouse model of Alzheimer's disease. Brain Res. 2013;1529:200-8.

10. Zhao HY, Wu HJ, He JL, Zhuang JH, Liu ZY, Huang LQ, et al. Chronic sleep restriction induces cognitive deficits and cortical beta-amyloid deposition in mice via BACE 1-antisense activation. CNS Neurosci Ther. 2017;23(3):233-40.

11. Ooms S, Overeem S, Besse K, Rikkert MO, Verbeek M, Claassen JA. Effect of 1 night of total sleep deprivation on cerebrospinal fluid $\beta$-amyloid 42 in healthy middle-aged men: a randomized clinical trial. JAMA neurology. 2014;71(8):971-7.

12. Ju Y-ES, Ooms SJ, Sutphen C, Macauley SL, Zangrilli MA, Jerome G, et al. Slow wave sleep disruption increases cerebrospinal fluid amyloid- $\beta$ levels. Brain. 2017;140(8):2104-11.

13. Shokri-Kojori E, Wang G-J, Wiers CE, Demiral SB, Guo M, Kim SW, et al. $\beta$ Amyloid accumulation in the human brain after one night of sleep deprivation. Proc Natl Acad Sci. 2018;115(17):4483-8.

14. Xie L, Kang H, Xu Q, Chen MJ, Liao Y, Thiyagarajan M, et al. Sleep drives metabolite clearance from the adult brain. Science (80- ). 2013; 342(6156):373-7.

15. Nliff JJ, Wang M, Liao Y, Plogg BA, Peng W, Gundersen GA, et al. A paravascular pathway facilitates CSF flow through the brain parenchyma and the clearance of interstitial solutes, including amyloid $\beta$. Sci Transl Med. 2012:4(147):147ra11-ra11.

16. Nedergaard M. Garbage truck of the brain. Science (80- ). 2013; 340(6140):1529-30.

17. Fultz NE, Bonmassar G, Setsompop K, Stickgold RA, Rosen BR, Polimeni JR, et al. Coupled electrophysiological, hemodynamic, and cerebrospinal fluid oscillations in human sleep. Science (80- ). 2019;366(6465):628-31.

18. Jessen NA, Munk ASF, Lundgaard I, Nedergaard M. The glymphatic system: a beginner's guide. Neurochem Res. 2015;40(12):2583-99.

19. Kang DW, Lee CU, Lim HK. Role of sleep disturbance in the trajectory of Alzheimer's disease. Clin Psychopharmacol Neurosci. 2017;15(2):89.

20. Lucey BP, Hicks TJ, McLeland JS, Toedebusch CD, Boyd J, Elbert DL, et al. Effect of sleep on overnight cerebrospinal fluid amyloid $\beta$ kinetics. Ann Neurol. 2018:83(1):197-204.

21. Faul F, Erdfelder E, Lang A-G, Buchner A. G* Power 3: a flexible statistical power analysis program for the social, behavioral, and biomedical sciences. Behav Res Methods. 2007;39(2):175-91.

22. Thomas J, Ooms S, Verbeek M, Booij J, Rijpkema M, Kessels RPC, et al. SleepCognition Hypothesis In maritime Pilots, what is the effect of long-term work-related poor sleep on cognition and amyloid accumulation in healthy middle-aged maritime pilots: methodology of a case-control study. BMJ Open. 2019;9(6):e026992

23. Garcia-Molina G, Tsoneva T, Jasko J, Steele B, Aquino A, Baher K, et al. Closed-loop system to enhance slow wave activity during sleep. 2018.

24. Weintraub S, Carrillo MC, Farias ST, Goldberg TE, Hendrix JA, Jaeger J, et al. Measuring cognition and function in the preclinical stage of Alzheimer's disease. Alzheimer's Dementia. 2018;4:64-75.

25. Baddeley A, Emslie H, Nimmo-Smith I. Doors and people: a test of visual and verbal recall and recognition, Thames Valley Test Company, Bury St. Edmunds, UK. 1994

26. Curtis C, Gamez JE, Singh U, Sadowsky CH, Villena T, Sabbagh MN, et al. Phase 3 trial of flutemetamol labeled with radioactive fluorine 18 imaging and neuritic plaque density. JAMA neurology. 2015;72(3):287-94.

27. Lowe VJ, Lundt E, Knopman D, Senjem ML, Gunter JL, Schwarz CG, et al. Comparison of [18F] Flutemetamol and [11C] Pittsburgh Compound-B in cognitively normal young, cognitively normal elderly, and Alzheimer's disease dementia individuals. Neurolmage: Clinical. 2017;16:295-302.

28. Hansson O, Seibyl J, Stomrud E, Zetterberg H, Trojanowski JQ, Bittner T, et al. CSF biomarkers of Alzheimer's disease concord with amyloid- $\beta$ PET and predict clinical progression: a study of fully automated immunoassays in BioFINDER and ADNI cohorts. Alzheimers Dement. 2018;14(11):1470-81.

29. Pannee J, Portelius E, Minthon L, Gobom J, Andreasson U, Zetterberg H, et al. Reference measurement procedure for CSF amyloid beta (A $\beta$ ) 1-42 and the CSF $A \beta 1-42 / A \beta 1-40$ ratio-a cross-validation study against amyloid PET. J Neurochem. 2016;139(4):651-8.

30. Palmqvist S, Zetterberg H, Blennow K, Vestberg S, Andreasson U, Brooks DJ, et al. Accuracy of brain amyloid detection in clinical practice using cerebrospinal fluid $\beta$-amyloid 42: a cross-validation study against amyloid positron emission tomography. JAMA neurology. 2014;71(10):1282-9. 
31. Palmqvist $\mathrm{S}$, Zetterberg $H$, Mattsson $N$, Johansson P, Minthon L, Blennow K, et al. Detailed comparison of amyloid PET and CSF biomarkers for identifying early Alzheimer disease. Neurology. 2015;85(14):1240-9.

32. Bouallègue FB, Mariano-Goulart D, Payoux P, Initiative AsDN. Comparison of CSF markers and semi-quantitative amyloid PET in Alzheimer's disease diagnosis and in cognitive impairment prognosis using the ADNI-2 database. Alzheimers Res Ther. 2017;9(1):32.

33. Reimand J, De Wilde A, Teunissen CE, Zwan M, Windhorst AD, Boellaard $R$, et al. PET and CSF amyloid- $\beta$ status are differently predicted by patient features: information from discordant cases. Alzheimers Res Ther. 2019;11(1):100.

34. Jenkinson M, Bannister P, Brady M, Smith S. Improved optimization for the robust and accurate linear registration and motion correction of brain images. Neuroimage. 2002;17(2):825-41.

35. Jenkinson M, Beckmann CF, Behrens TE, Woolrich MW, Smith SM. Fs Neuroimage. 2012;62(2):782-90.

36. Andersson $\mathrm{J}$, Jenkinson $\mathrm{M}, \mathrm{Smith} \mathrm{S}$. Non-linear registration aka Spatial normalisation FMRIB Technial Report TR07JA2. FMRIB Analysis Group of the University of Oxford 2007.

37. Farrar $\mathrm{G}$, Molinuevo $\mathrm{J}$, Zanette $\mathrm{M}$. Is there a difference in regional read [18 F] flutemetamol amyloid patterns between end-of-life subjects and those with amnestic mild cognitive impairment? Eur J Nucl Med Mol Imaging. 2019;46(6):1299-308.

38. Vandenberghe R, Van Laere K, Ivanoiu A, Salmon E, Bastin C, Triau E, et al. 18F-flutemetamol amyloid imaging in Alzheimer disease and mild cognitive impairment: a phase 2 trial. Ann Neurol. 2010;68(3):319-29.

39. Thurfjell L, Lötjönen J, Lundqvist R, Koikkalainen J, Soininen H, Waldemar G, et al. Combination of biomarkers: PET [18F] flutemetamol imaging and structural MRI in dementia and mild cognitive impairment. Neurodegener Dis. 2012;10(1-4):246-9.

40. Buckley CJ, Sherwin PF, Smith AP, Wolber J, Weick SM, Brooks DJ. Validation of an electronic image reader training programme for interpretation of [18F] flutemetamol $\beta$-amyloid PET brain images. Nucl Med Commun. 2017;38(3):234

41. Winer JR, Mander BA, Helfrich RF, Maass A, Harrison TM, Baker SL, et al. Sleep as a potential biomarker of tau and $\beta$-amyloid burden in the human brain. J Neurosci. 2019:0503-19.

42. Lucey BP, McCullough A, Landsness EC, Toedebusch CD, McLeland JS, Zaza $A M$, et al. Reduced non-rapid eye movement sleep is associated with tau pathology in early Alzheimer's disease. Sci Transl Med. 2019;11(474): eaau6550.

43. Mander BA, Marks SM, Vogel JW, Rao V, Lu B, Saletin JM, et al. [beta]amyloid disrupts human NREM slow waves and related hippocampusdependent memory consolidation. Nat Neurosci. 2015;18(7):1051-7.

44. Jansen WJ, Ossenkoppele R, Knol DL, Tijms BM, Scheltens P, Verhey FR, et al. Prevalence of cerebral amyloid pathology in persons without dementia: a meta-analysis. Jama. 2015;313(19):1924-38

45. Kuehn BM. In Alzheimer Research. JAMA: Glucose metabolism moves to center stage; 2020.

46. Smith MA, Rottkamp CA, Nunomura A, Raina AK, Perry G. Oxidative stress in Alzheimer's disease. Biochimica et Biophysica Acta (BBA)-Molecular Basis of Disease. 2000;1502(1):139-44.

47. Green RC, Cupples LA, Kurz A, Auerbach S, Go R, Sadovnick D, et al. Depression as a risk factor for Alzheimer disease: the MIRAGE Study. Arch Neurol. 2003;60(5):753-9.

48. Rabin JS, Klein H, Kirn DR, Schultz AP, Yang HS, Hampton O, Pruzin J. Associations of physical activity and $\beta$-amyloid with longitudinal cognition and neurodegeneration in clinically normal older adults. JAMA neurology. 2019:76(10):1203-10.

49. Lysen TS, Wolters FJ, Luik Al, Ikram MK, Tiemeier H, Ikram MA. Subjective sleep quality is not associated with incident dementia: the Rotterdam study. J Alzheimers Dis. 2018;64(1):239-47.

50. Holth JK, Fritschi SK, Wang C, Pedersen NP, Cirrito JR, Mahan TE, et al. The sleep-wake cycle regulates brain interstitial fluid tau in mice and CSF tau in humans. Science (80- ). 2019;363(6429):880-4.

51. Bejanin A, Schonhaut DR, La Joie R, Kramer JH, Baker SL, Sosa N, et al. Tau pathology and neurodegeneration contribute to cognitive impairment in Alzheimer's disease. Brain. 2017;140(12):3286-300.

52. Pontecorvo MJ, Devous MD Sr, Navitsky M, Lu M, Salloway S, Schaerf FW, et al. Relationships between flortaucipir PET tau binding and amyloid burden, clinical diagnosis, age and cognition. Brain. 2017;140(3):748-63.
53. Ossenkoppele R, Smith R, Ohlsson T, Strandberg O, Mattsson N, Insel PS, et al. Associations between tau, $A \beta$, and cortical thickness with cognition in Alzheimer disease. Neurology. 2019;92(6):e601-e12.

\section{Publisher's Note}

Springer Nature remains neutral with regard to jurisdictional claims in published maps and institutional affiliations.
Ready to submit your research? Choose BMC and benefit from:

- fast, convenient online submission

- thorough peer review by experienced researchers in your field

- rapid publication on acceptance

- support for research data, including large and complex data types

- gold Open Access which fosters wider collaboration and increased citations

- maximum visibility for your research: over $100 \mathrm{M}$ website views per year

At BMC, research is always in progress.

Learn more biomedcentral.com/submissions 